\title{
VARIAÇÕES FISIOLÓGICAS DE CÃES ENVOLVIDOS EM ATIVIDADES ASSISTIDAS E AVALIAÇÃO DO DIAGNÓSTICO DE SATISFAÇÃO DOS PROPRIETÁRIOS
}

\author{
Helena Magalhães Ferreira, Juliana Samila de Castro Miguel, Maria Luiza Poiatti
}

Universidade Estadual Paulista "Júlio de Mesquita Filho", curso de Zootecnia, Dracena, SP. E-mail: helenamagalhaes@hotmail.com

\section{RESUMO \\ Nas atividades Assistidas por Animais (AAA) os animais atuam como elo para estimular a sociabilidade do homem. O objetivo deste estudo foi avaliar os efeitos das atividades assistidas, através da medição de parâmetros fisiológicos de 15 cães co-terapeutas utilizados nas sessões de AAA junto à APAE de Dracena. Foi realizada uma avaliação de diagnóstico com os proprietários dos cães, para medir o grau de satisfação atribuído ao desenvolvimento do projeto de extensão Cão Cidadão Unesp de Dracena. Houve diferença significativa entre portes, onde as médias dos cães de médio porte se apresentaram superiores aos de pequeno porte. As diferentes situações também apresentaram diferença significativa, essas alterações foram atribuídas devido à excitação dos animais, manipulação, presença de outros cães e pessoas. Os cães não apresentaram estresse negativo. Considerando o questionário de satisfação dos proprietários, as AAA são benéficas à saúde de seus cães, resultando em um alto grau de satisfação. \\ Palavras-chave: Atividades Assistidas por Animais, Avaliação Fisiológica em Cães, Cães Co- terapeutas \\ PHYSIOLOGICAL VARIATIONS OF DOGS INVOLVED IN ASSISTED-ACTIVITIES AND ASSESSMENT OF THE DIAGNOSIS OF OWNERS SATISFACTION}

\section{ABSTRACT}

In Animal-Assisted Activities (AAA), the animals act as a link to stimulate the sociability of man. The aim of this study was to evaluate the effects of assisted-activities, through measuring the physiological parameters of 15 co-therapists dogs used in the AAA sessions, along with the APAE of Dracena. Was performed a diagnostic evaluation with the dog owners, to measure the degree of satisfaction attributed to the development of the extension project "Cão Cidadão Unesp de Dracena"There was a significant difference between sizes, where the averages of the mediumsized dogs were superior to the small size ones. The different situations also presented significant difference, these alterations were attributed due the excitation of the animals, manipulation, presence of other dogs and people. The dogs did not present negative stress. Considering the owners satisfaction questionnaire, the AAAs are beneficial to the health of their dogs, resulting in a high degree of satisfaction.

Keywords: Animal-Assisted Activities, Physiological Evaluation in Dogs, Dogs Co-therapists

\section{INTRODUÇÃO}

As Atividades Assistidas por Animais (AAA) são aquelas que visam, de maneira informal, a obtenção de benefícios motivacionais, educacionais e recreativos, contribuindo para um aumento na qualidade de vida. Podendo ser realizadas em diversos ambientes sob a responsabilidade de profissionais ou de voluntários credenciados que atuam em conjunto com animais selecionados (Delta Society, 1996). Berzins (2000) observou que a presença do animal auxilia na redução de 
problemas como a ansiedade, o estresse e alterações cardíacas, em que a recuperação se tornou mais rápida e com maior ênfase até mesmo na depressão, pelo simples fato das pessoas levarem seus companheiros para passear.

Segundo Leal e Natalie (2007) as atividades que utilizam a participação de animais como a AAA possuem objetivos diretos de promover atividades que contribuam para a saúde e o bemestar dos indivíduos, tanto com função motivacional, educacional, lúdica ou terapêutica, assim como o de melhorar o funcionamento físico, social, emocional e cognitivo.

Considerando os trabalhos de vários autores e no desenvolvimento das atividades do projeto de extensão Cão Cidadão da Unesp de Dracena, realizado na instituição de ensino APAE, para a melhoria de qualidade de vida das pessoas com síndromes, necessidades especiais e autistas, é que houve o interesse em investigar as variações fisiológicas e benefícios ligados aos cães durante as AAA. Assim como o interesse sobre a avaliação de satisfação dos proprietários dos cães envolvidos no projeto.

\section{METODOLOGIA}

O presente trabalho foi aprovado na CEUA - Comissão de Ética no Uso de Animais, registrado com o protocolo 28/2016, na FCAT - Unesp de Dracena.

Semanalmente, uma equipe composta por quinze alunos do curso de Zootecnia da Faculdade de Ciências Agrárias e Tecnológicas - UNESP Campus de Dracena e uma Médica Veterinária, desenvolveram as AAA na Associação de Pais e Amigos dos Excepcionais - APAE de Dracena. Essas atividades são derivadas do projeto de extensão Cão Cidadão - Unesp de Dracena, que desde o ano de 2011 realiza múltiplas atividades assistidas com os educandos da APAE, local que recebe aproximadamente duzentos alunos (crianças e adultos) com diferentes tipos de síndromes diariamente, para realização de diversas atividades escolares e atendimento médico.

A realização das AAA ocorreu nos meses de agosto a outubro de 2016, às quartas-feiras no período da tarde, das $14 \mathrm{~h}$ às $15 \mathrm{~h} 30$, de maneira contínua, sendo interrompidas apenas em decorrência de feriados ou imprevistos (falta de transporte, eventos acadêmicos ou doenças de cães), totalizando 10 visitas na (APAE) e nas residências. As atividades duraram em média 90 minutos e, na maioria delas foram utilizados 5 cães por visita, sendo três cães da raça Border Collie, três Australian Cattle dogs, um Golden Retriever, um Lhasa Apso, dois Labrador, um Pug, um Shitzu, um Tekel e dois SRD (Sem Raça Definida), totalizando 15 cães. Todos os cães utilizados são pertencentes aos colaboradores do projeto de extensão Cão Cidadão - Unesp de Dracena, entre alunos, docentes da Unesp Dracena e moradores da cidade. Foram utilizados animais não adestrados e, para que não houvesse algum problema nas sessões, foi realizada uma seleção prévia dos animais e escolhidos apenas aqueles com características dóceis, que tivessem afinidade com crianças, ausência de medo e de reações violentas. A seleção de animais aptos a esse tipo de intervenção foi de grande importância, devido à estimulação dos alunos à recreação, como tocar e afagar a pelagem, atividades de entretenimento, assim como caminhadas e corridas com os cães.

$\mathrm{Na}$ presente pesquisa houve a divisão em três diferentes situações: S1 controle (sem desenvolvimento das atividades assistidas, situação de repouso em seu ambiente domiciliar; S2 (antes do início das atividades assistidas, já no local das atividades); e S3 (logo após o término das atividades assistidas)). Para cada situação foram aferidas cinco variáveis: Frequência Cardíaca (FC), Frequência Respiratória (FR), Temperatura Retal (TR), Pressão Arterial Sistólica (PAS) e Pressão Arterial Diastólica (PAD). Todas as aferições foram medidas pela Médica Veterinária Juliana Andréa Osorio Balan, colaboradora do projeto.

A aferição da FC foi realizada por meio de auscultação, com o auxílio de um estetoscópio (Figura 1), que foi ajustado no peitoral dos cães e os batimentos cardíacos foram contados por 15 
segundos, em seguida o valor obtido foi multiplicado por 4. Este método é utilizado comumente em cães mais agitados, sendo a melhor forma para diminuir o tempo de imobilização dos mesmos.

A FR também foi aferida com o auxílio do estetoscópio, contando os movimentos abdominais por 15 segundos e multiplicado o valor obtido por 4 .

A TR foi aferida por meio de um termômetro digital para o uso veterinário (Figura 2), sendo introduzido no reto dos cães e mensurado o resultado, sendo considerado o único método de aferição invasivo.

A PAS e PAD foram aferidas por meio de um esfigmomanômetro digital de uso veterinário (Figura 3), sendo ajustado à um dos membros anteriores dos cães, onde encontra-se o vaso sanguíneo mais utilizado para esta aferição, denominada cefálica.
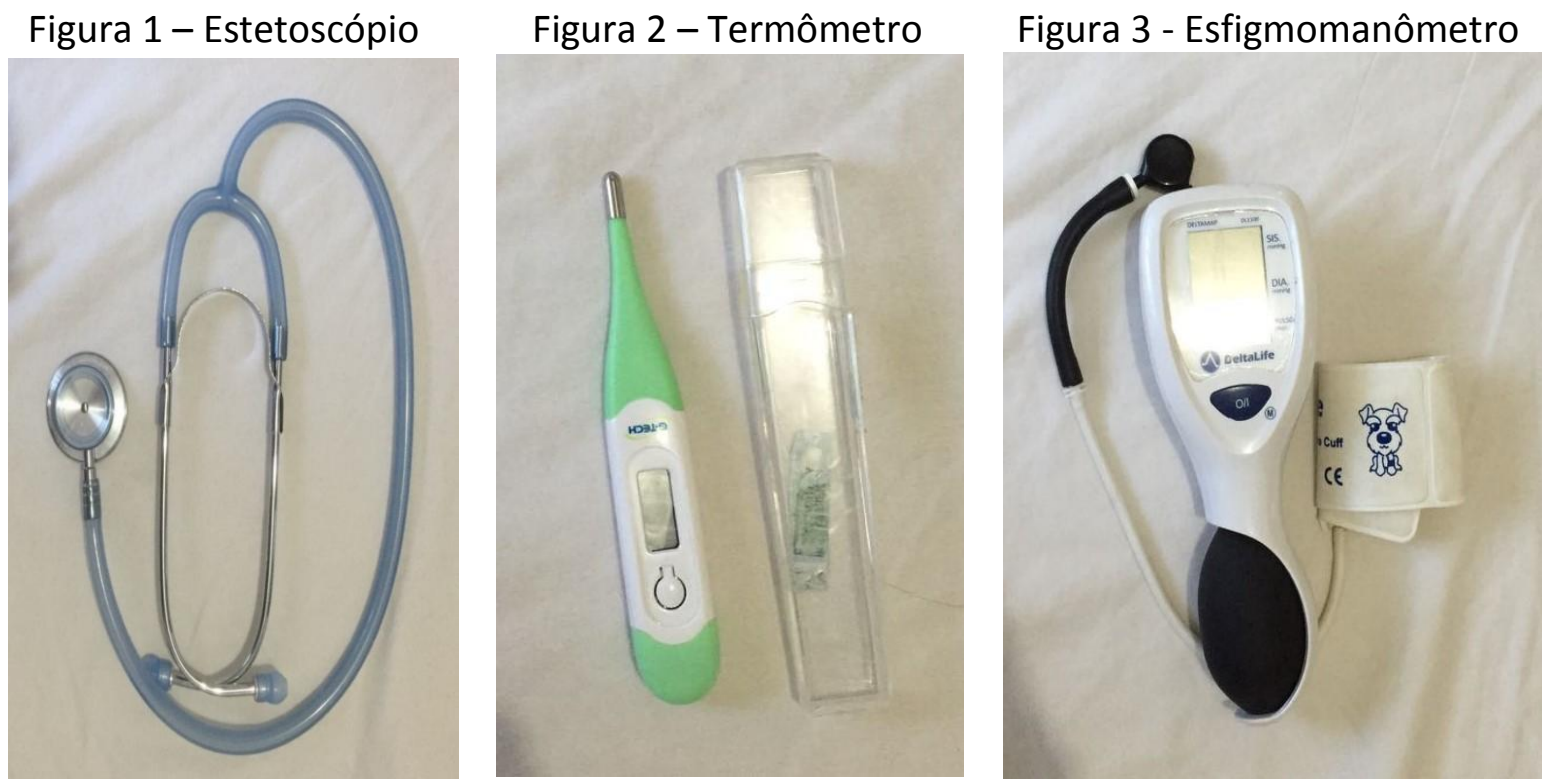

Fonte: Arquivo Pessoal.

Além das aferições das variáveis fisiológicas, foi realizada uma avaliação do diagnóstico de satisfação dos proprietários em relação as respostas comportamentais de seus cães quando estão desenvolvendo as AAA, por meio de um questionário adaptado da literatura de Yazbek e Fantoni (2005) com 10 questões.

O delineamento experimental foi inteiramente casualizado em esquema fatorial $3 \times 2$. Considerou-se no fator $A$ as três situações (S1, S2 e S3) e no fator B os portes dos cães (pequeno e médio porte). Foi aplicado o método dos mínimos quadrados para estimação dos parâmetros do modelo estatístico descrito abaixo.

O modelo estatístico e pressuposições:

$$
Y i j k=\mu+\tau i+\beta j+(\tau \beta) i j+\varepsilon i j k
$$

Yijk é o valor observado para a variável em estudo referente ao i-ésimo nível do fator $A$ com o jésimo nível do fator $B$;

$\mu$ é a média de todas as unidades experimentais para a variável em estudo;

ti é o efeito do i-ésimo nível do fator;

ßj é o efeito do j-ésimo nível do fator $B$;

$(\tau \beta)$ ij é o efeito da interação do i-ésimo nível do fator $A$ com o j-ésimo nível do fator $B$;

عijk é o erro aleatório associado à observação.

Em que $\tau i, \beta j,(\tau \beta) i j$ e $\varepsilon i j k$, são variáveis aleatórias independentes assumindo distribuição normal com média zero e variância constante (PIMENTEL-GOMES, 2009). 


\section{RESULTADOS}

Na tabela 1 estão apresentados os resultados das interações de FC, FR, TR, PAS e PAD. Verificou-se que não houve efeito significativo para as interações entre os fatores principais; assim pode-se afirmar que não houve dependência entre os fatores Porte e Situações. Para o fator Porte, verificou-se que o teste foi significativo $(P<0,05)$, indicando que o diferente Porte dos cães (pequeno e médio) apresentou efeitos diferentes sobre as variáveis frequência cardíaca, pressão arterial sistólica e pressão arterial diastólica. Para o fator Situações, verificou-se que o teste é significativo $(P<0,05)$, indicando que as diferentes situações de mensurações nos cães (situação 1 , situação 2 e situação 3 ) apresentaram efeitos diferentes sobre as variáveis: frequência cardíaca, frequência respiratória e temperatura retal.

O teste Tukey (Tabela 2 ) para comparações das médias ( $5 \%$ de significância) foi aplicado nas variáveis em que a análise de variância foi significativa $(P<0,05)$. As médias dos cães de porte médio são significativamente superiores às obtidas para o porte pequeno com relação as variáveis frequência cardíaca, pressão arterial sistólica e pressão arterial diastólica. Já as médias referentes a situação 3 são significativamente superiores às obtidas para as outras situações com relação as variáveis frequência cardíaca, frequência respiratória e temperatura retal.

Tabela 1 - P-valor referente a análise de variância de frequência cardíaca (FC); frequência respiratória (FR); temperatura retal (TR); pressão arterial sistólica (PS) e pressão diastólica (PD) de cães submetidos às Atividades Assistidas no período de agosto a outubro de 2016.

\begin{tabular}{cccccc}
\hline & FC & FR & TR & PS & PD \\
\hline Porte & $0,0460^{*}$ & 0,1924 & 0,205 & $0,0003^{*}$ & $0,0173^{*}$ \\
\hline Situaçóes & $0,0444^{*}$ & $0,0377^{* *}$ & $1,68 \mathrm{e}-05^{* * *}$ & 0,6968 & 0,6731 \\
\hline $\begin{array}{c}\text { Porte } x \\
\text { Situaçóes }\end{array}$ & 0,7625 & 0,9709 & 0,544 & 0,9016 & 0,2089 \\
\hline
\end{tabular}

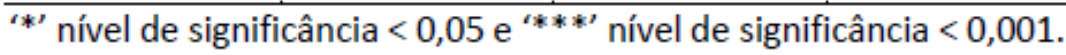


Tabela 2 - Médias referentes a análise de variância de frequência cardíaca (FC); frequência respiratória (FR); temperatura retal (TR); pressão arterial sistólica (PS) e pressão diastólica (PD) de cães submetidos as Atividades Assistidas no período de agosto a outubro de 2016.

\begin{tabular}{cccccc}
\hline & FC & FR & TR & PS & PD \\
\hline \multicolumn{7}{c}{ Portes dos cães } \\
\hline Médio & $121,10 \mathrm{a}$ & 140,6 & 39,09 & $155,82 \mathrm{a}$ & $102,65 \mathrm{a}$ \\
\hline Pequeno & $101,06 \mathrm{~b}$ & 101,9 & 38,81 & $124,6 \mathrm{~b}$ & $83,46 \mathrm{~b}$ \\
\hline $\mathbf{8}$ & \multicolumn{6}{c}{ Situações } \\
\hline S1 & $128,78 \mathrm{a}$ & $181,13 \mathrm{a}$ & $39,6 \mathrm{a}$ & 149,93 & 99,66 \\
\hline S2 & $114,6 \mathrm{ab}$ & $139,35 \mathrm{ab}$ & $38,26 \mathrm{~b}$ & 143,13 & 97,06 \\
\hline
\end{tabular}

Obs: Médias seguidas pela mesma letra nas colunas não diferem estatisticamente pelo teste Tukey

$5 \%$ de probabilidade.

Houve diferença significativa entre as situações. Com relação a FC, houve variação entre as médias nas situações e os portes, apresentando elevação significativa em S3 e porte médio, a qual pode ser decorrente das atividades exercidas durante as AAA, como a corrida para os cães de porte médio e apenas caminhada e afagos para os cães de pequeno porte.

Houve diferença significativa dos valores obtidos da temperatura na S3, em razão provavelmente, da manipulação e do transporte dos animais até o local e das atividades durante as AAA.

Tabela 3 - Questionário aplicado aos proprietários dos cães que foram utilizados na AAA.

\begin{tabular}{|c|c|c|}
\hline Perguntas & Respostas & Total $(\%)$ \\
\hline AAA atrapalham a vida do seu cäo? & Năo & $100 \%$ \\
\hline $\begin{array}{l}\text { Seu animal continua fazendo o que mais } \\
\text { gosta após ingresso na AAA? }\end{array}$ & Normalmente & $100 \%$ \\
\hline \multirow{3}{*}{$\begin{array}{l}\text { O temperamento do seu cão mudou após } \\
\text { ingresso na AAA? }\end{array}$} & Mudou pouco & $20 \%$ \\
\hline & Normal & $80 \%$ \\
\hline & $\begin{array}{l}\text { Fica um pouco mais } \\
\text { cansado que o normal }\end{array}$ & $13,33 \%$ \\
\hline \multirow[t]{2}{*}{ Ao retornar das AAA o seu animal: } & Fica normal & $13,33 \%$ \\
\hline & $\begin{array}{l}\text { Fica alegre e bem mais } \\
\text { disposto }\end{array}$ & $73,34 \%$ \\
\hline
\end{tabular}

\begin{tabular}{|c|c|c|}
\hline \multirow{3}{*}{ Ao retornar das AAA o seu animal: } & Se alimenta normalmente & $73,34 \%$ \\
\hline & $\begin{array}{l}\text { Ingere uma quantidade } \\
\text { superior }\end{array}$ & $20 \%$ \\
\hline & Recusa em alimentar-se & 6,66 \\
\hline
\end{tabular}




\begin{tabular}{llc}
\hline \multirow{2}{*}{ Ao perceber que vai para a AAA o seu animal: } & \multicolumn{1}{l}{ Fica alegre } & $73,34 \%$ \\
\cline { 2 - 3 } & Fica eufórico & $20 \%$ \\
\cline { 2 - 3 } & Fica indiferente & 6,66 \\
\hline $\begin{array}{l}\text { Dificuldade de locomoção após ingresso na } \\
\text { AAA? }\end{array}$ & $\begin{array}{l}\text { Não apresentam } \\
\text { dificuldade }\end{array}$ & $100 \%$ \\
\hline & Raramente & $60,01 \%$ \\
\cline { 2 - 3 } & Frequentemente & $20 \%$ \\
\cline { 2 - 3 } Seu animal se cansa facilmente? & Está normal & $13,33 \%$ \\
\cline { 2 - 3 } & Sempre & $100 \%$ \\
\hline $\begin{array}{l}\text { Seu cão passou a assustar-se facilmente após } \\
\text { a AAA? }\end{array}$ & Não houve diferença & \\
\hline
\end{tabular}

Gráfico 1 - Satisfação dos proprietários dos cães submetidos a AAA.

Satisfação dos proprietários dos cães submetidos a AAA.

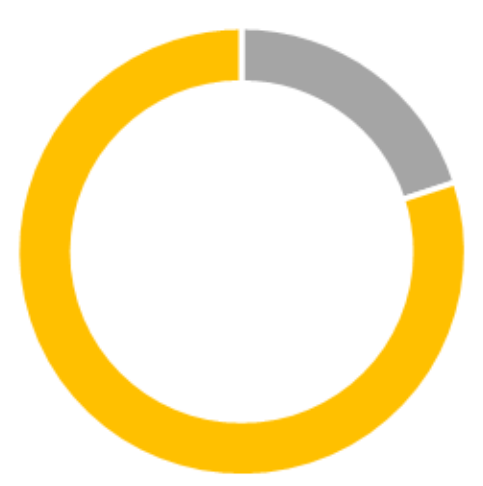

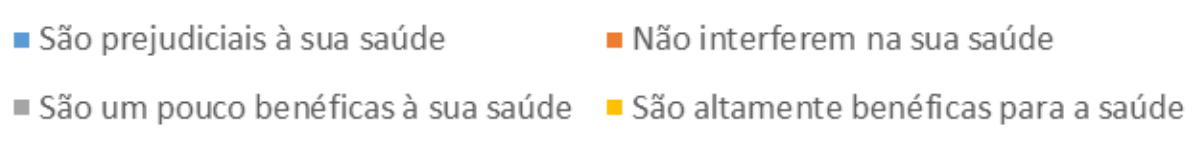

Fonte: Arquivo Pessoal.

\section{DISCUSSÃO}

A FR atingiu valores considerados acima da normalidade para todos os cães avaliados, em todas as situações e portes. A elevação da frequência respiratória em S3 provavelmente é atribuída à excitação do cão pelo contato com os alunos da APAE, bem como à manipulação e contenção do animal ao término das AAA.

Os valores de PS e PD encontraram-se dentro da normalidade para os cães de porte pequeno, porém com média elevada nos cães de porte médio. Tal fato pode estar associado à maior intensidade nas atividades exercidas durante as AAA.

Foi excluída a possibilidade de a elevação da temperatura ser indicativa de alguma alteração sistêmica patológica, tendo em vista que os animais não desenvolveram sintomas de nenhuma 
doença. Essa alteração foi, portanto, atribuída ao clima extremamente quente da cidade, ao exercício e à excitação decorrente da atividade desenvolvida. Houve uma variação na temperatura ambiental (entre 24 e $36^{\circ} \mathrm{C}$ ) entre os dias em que as aferições foram realizadas.

Yamamoto (2012) aferiu a temperatura retal, a pressão arterial sistólica e as frequências cardíaca e respiratória nos seguintes momentos: M0 (média de três avaliações do cão em repouso) e em M1, M2 e M3 (imediatamente antes, imediatamente após e decorridas 24h das atividades), porém não encontrou diferença significativa quanto às características estudadas $(P>0,05)$. Haubenhofer et al. (2006) relata possíveis causas de ansiedade nos cães, diretamente relacionadas a alterações fisiológicas das variáveis em estudo, destacando: o transporte, contato com outros cães, contato com pessoas desconhecidas e novo ambiente.

\section{CONCLUSÃO}

Na presente pesquisa foi possível concluir que os cães envolvidos em atividades assistidas apresentaram diferença significativa de variações fisiológicas entre médio e pequeno porte, sendo consideradas respostas ao estímulo imediato (em curto prazo).

Os resultados obtidos podem estar relacionados com a atividade específica que cada cão pratica nas AAA. Nenhum cão apresentou comportamento de estresse negativo.

Considerando o questionário de satisfação dos proprietários, conclui-se que as AAA são altamente benéficas à saúde de seus cães, resultado em um alto grau de satisfação dos proprietários.

\section{REFERÊNCIAS}

Berzins, Marilia A. V. da Silva. Velhos, cães e gatos: interpretação de uma relação. Dissertação de Mestrado em Gerontologia. São Paulo: PUC-SP, 2000.

Delta Society. Standads of Practice for Animal-Assisted Activities and Animal-Assisted Therapy Renton. WA: Delta Society, 1996.

Haubenhofer, D.K.; Kirchengast, S. Physiological arousal for companion dogs working with their owners in animal-assisted activities and animal-assisted therapy. J. Appl. Anim. Welf. Sci. v.9, 2006. p.165-172. https://doi.org/10.1207/s15327604jaws0902 5

Leal, G.; Natalie, K. Afeto que cura. Disponível em: www.fag.edu.br/graduacao/fisioterapia/arquivos/afetoquecura.pdf Acesso em: 25 de outubro de 2016.

Yamamoto, K.C.M. Avaliação fisiológica e comportamental de cães utilizados em terapia assistida por animais (TAA), 2012. 